\title{
The $n$-person Kalai-Smorodinsky bargaining solution under pre-donations
}

\author{
S. Nuray Akin • Brennan C. Platt • \\ Murat R. Sertel
}

Received: 29 July 2009 / Accepted: 9 October 2010 / Published online: 27 October 2010 (C) Springer-Verlag 2010

\begin{abstract}
This study examines the behavior of simple $n$-person bargaining problems under pre-donations where the Kalai-Smorodinsky (KS) solution is operant. Predonations are a unilateral commitment to transfer a portion of one's utility to someone else, and are used to distort the bargaining set and thereby influence the bargaining solution. In equilibrium, these pre-donations are Pareto-improving over the undistorted solution; moreover, when the agents' preferences are sufficiently distinct, the equilibrium solution coincides with the concessionary division rule.
\end{abstract}

Keywords Bargaining $\cdot$ Concession $\cdot$ Pre-donation $\cdot$ Kalai-Smorodinsky solution

JEL Classification $\quad$ C7 $\cdot$ D7

Murat R. Sertel deceased in 2003. Much of this paper was written when the corresponding author was a graduate student in Bogazici University, Istanbul, Turkey (Akin 2001) and later at the University of Minnesota, MN, USA. Sertel was a Professor of Economics in Bogazici University and Bilkent University in Turkey. We honor his contributions to economic theory and his role as a mentor to many students throughout his career.

\section{S. N. Akin $(\varangle)$}

Department of Economics, University of Miami, 517-K Jenkins, Coral Gables, FL 33124, USA

e-mail: nakin@miami.edu

B. C. Platt

Department of Economics, Brigham Young University, 135 FOB, Provo, UT 84602, USA

e-mail: brennan_platt@byu.edu

M. R. Sertel

Department of Economics, Bogazici University, Istanbul, Turkey

M. R. Sertel

Department of Economics, Bilkent University, Ankara, Turkey 


\section{Introduction}

Consider a perfectly divisible good that needs to be allocated among several individuals. Each agent receives a constant marginal utility $\alpha_{i}$ per unit of the good, which may differ across agents. As in Sertel $(1991,1992)$, this choice of allocation can be analyzed as a bargaining problem, where the bargaining set indicates all of the possible utility vectors from any particular division of the good and the threat point is the origin.

Suppose that the outcome is to be determined by a particular bargaining solution. A bargaining solution takes the bargaining set as its domain and selects a particular utility vector from the set as the outcome. Thus, could the individuals improve their outcomes in the bargaining solution by somehow distorting the underlying bargaining set?

Here, we examine how the Kalai-Smorodinsky (KS) bargaining solution (Kalai and Smorodinsky 1975; Kalai 1977, 1985) can be distorted using pre-donations. A pre-donation is a binding, unilateral commitment by an agent to transfer a fraction of his utility to another agent, via some numeraire good for which all agents have equal marginal utility. These commitments are simultaneously chosen by the agents; the KS solution is then applied to the distorted bargaining set.

We show that pre-donations will occur in equilibrium if the marginal utilities $\alpha$ are sufficiently distinct. Moreover, when they occur, pre-donations are in fact Pareto improving, helping the donor, the recipient, and all others. This somewhat surprising result arises because pre-donations transfer utility more effectively than changing the allocation of the good. The original bargaining problem cannot impose transfers among the agents, but only split the good in question. Pre-donations will distort the bargaining solution so that a larger portion of the good is allocated to the agent with the highest $\alpha$, who then compensates the others via pre-donations.

In particular, if $\alpha_{i} \geq 2 \alpha_{i-1}$ for all $i$ (where agents are labelled in order of increasing marginal utility), then the good is fully allocated to agent $n$, who transfers $\alpha_{n-1}$ to agent $n-1$. He, in turn, donates $\alpha_{n-2}$ to agent $n-2$, and so on. Thus each agent $i$ receives a payoff $\alpha_{i}-\alpha_{i-1}$, which is to say, the payoff as if he had been allocated the full good, minus compensation given to the agent immediately below him. This coincides with the Concessionary Division rule, discussed in Sect. 5.

Our paper is most closely related to Sertel (1992), which examines the two-person Nash bargaining solution (Nash 1950) under pre-donations. He shows that only the agent with a higher $\alpha$ makes a pre-donation, and that when the Nash solution is applied to the distorted bargaining set, the outcome coincides with the Talmudic division rule. Sertel and Orbay (1998) analyzed the same question allowing for an arbitrary threat point rather than restricting it to the origin. Orbay (2003) also examines two-person bargaining problems with pre-donations and arbitrary threat point. Under both the KS solution as well as the Maschler-Perles solution (Maschler and Perles 1981), she finds that there is almost always an incentive to the agent with a higher marginal utility to make a pre-donation. ${ }^{1}$

\footnotetext{
1 When the threat point is set to 0 in Orbay, her results coincide with our two-person case, as does Sertel (1991). When the threat point is strictly positive, the agent with higher marginal utility has greater incentive to pre-donate, but this still only results in an efficient allocation if the marginal utilities are sufficiently distinct, as in our work.
} 
This paper also relates to a larger class of mechanism design problems in which agents can profitably manipulate a solution concept. The most well-known manipulation mechanism is misrepresentation of utility functions by agents in an exchange economy with a competitive allocation: in order to achieve a better outcome for himself, an agent can behave as if his utility function is different than the true one. Hurwicz (1972) shows that any Pareto optimal and individually rational reallocation scheme suffers from this problem. Another well-known mechanism is manipulation via hiding, transfer, or destruction of endowments. Some examples of this are resource reallocation mechanisms (Postlewaite 1974), Lindahl Equilibria (Sertel 1994), the men- or women-optimal matching rule (Sertel and Sanver 2002), and exchange markets with indivisible goods (Atlamaz and Klaus 2007).

Even so, the distortion of bargaining solutions does differ from manipulation in classic mechanism design. There, agent types are unknown to the designer, who must check whether agents would benefit by pretending to be a different type. Our environment takes place with perfect information: the marginal utilities of the individuals, the bargaining set, and the bargaining solution are all common knowledge. Indeed, the pre-donations ${ }^{2}$ have an obvious effect on the bargaining set, which the implementor of the solution concept could readily invert. Moreover, while manipulations often hinder the efficiency of a mechanism, distortions offer an improvement in our environment.

In addition to the problem of allocating a divisible good, this work can be readily applied to other interesting economic problems. Orbay (2003) suggests a cartel's problem of dividing a market among its members, where one firm is more efficient than the other and thus has strictly higher marginal profit. The undistorted KS solution would equally divide the market in order to satisfy the less efficient firm, even though it results in lower total profits for the cartel. Allowing pre-donations, however, would result in the more efficient firm serving the entire market, while compensating the less efficient firm as if he had been the only firm operating. Our work shows similar results in an $n$ firm cartel.

Our model also has applications in finance. For example, when a business venture falls into default, a distribution problem occurs where the creditors have to be compensated with the available assets. However, during market downturns, it is often the case that there is no immediate buyer for the property, making the value of the assets uncertain. One of the claimants might be willing to sell it at a very low price; but the other may be more patient, preferring to let the asset recover its value before being liquidated. The undistorted KS solution would result in equal division, but predonations would enable the most patient creditors to retain the assets while compensating those eager to liquidate.

We proceed as follows: Sect. 2 provides definitions and presents the model. Section 3 examines the model in the three-person case, providing illustration and intuition for the $n$-person case presented in Sect. 4. Section 5 discusses an alternative interpretation of our bargaining problem as a division problem (as in Sertel 1992) and proves that the pre-donation equilibrium coincides with the outcome of the Concessionary Division rule under certain conditions. Section 6 concludes.

\footnotetext{
2 Sertel (1992) called pre-donations a manipulation of the bargaining set, but to avoid confusion with the mechanism design literature, we have employed the term distortion instead.
} 

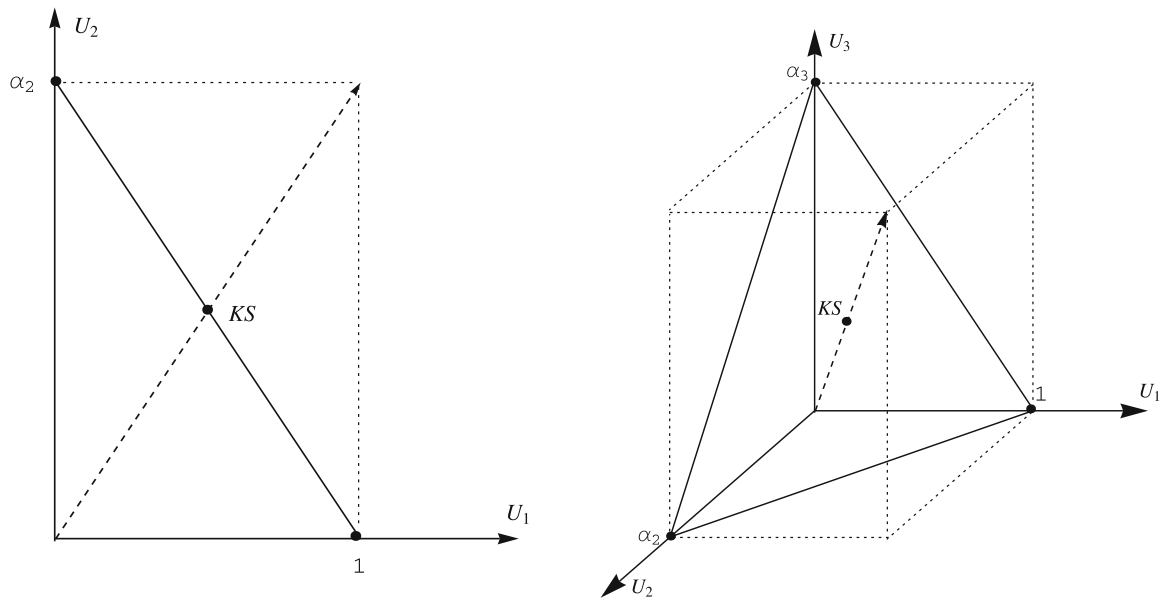

Fig. 1 Two- and three-person simple bargaining sets, and their KS solution

\section{Model}

Let $N=\{1,2, \ldots, n\}$ be the set of agents. Given any integer $n \geq 2$, an $n$-person bargaining problem is an ordered pair $(S, d)$, where $S$ is a compact and convex subset of $\mathbb{R}^{n}, d \in S$, and there exists $u \in S$ such that $u_{i}>d_{i}$ for each $i \in N$. Here, $S$ is called the bargaining set (or feasible set) and consists of all the utility vectors that can be attained if the bargainers mutually agree. $d$ is called the disagreement or threat point, which describes the utility vector if bargainers cannot reach an agreement.

Following Sertel (1992), we set $d=0$, the origin of $\mathbb{R}^{n}$; thus, the bargaining problem is determined by its bargaining set only. We also restrict ourselves to the following class of bargaining problems.

Definition 1 A bargaining problem is called simple if and only if it has a bargaining set of the form:

$$
S_{\alpha} \equiv\left\{u \in \mathbb{R}_{+}^{n} \mid u_{n} \leq \alpha_{n} \text { and } u_{k} \leq \alpha_{k}\left(1-\sum_{i=k+1}^{n} \frac{u_{i}}{\alpha_{i}}\right) \text { for all } k \leq n-1\right\}
$$

where $\alpha \in \mathbb{R}_{+}^{n}$ and $1=\alpha_{1}<\alpha_{2}<\cdots<\alpha_{n}$.

For example, in the two-person case, the feasible set is a triangular area in the non-negative orthant of the two-dimensional plane with a unit base and a height of $\alpha_{2}$. For three agents, this extends to a tetrahedron of height $\alpha_{3}$ along the $U_{3}$ axis; both are illustrated in Fig. 1.

If one interprets the bargaining problem as allocating a divisible good among the $N$ agents, a simple bargaining set means that agent $k$ receives constant marginal utility $\alpha_{k}$ from a unit of the good. The frontier of the set is a hyperplane whose slope between any agent $i$ and $j$ is the ratio of their marginal utilities: $-\frac{\alpha_{j}}{\alpha_{i}}$. 
If $B$ denotes a class of bargaining problems, a bargaining solution is a function $f: B \rightarrow \mathbb{R}^{n}$ that assigns a unique outcome $f(S, d) \in S$ for any bargaining set $(S, d) \in B$. These are usually axiomatically derived, and are an attempt to identify a reasonable outcome of the process of bargaining, without explicitly modeling that process in a non-cooperative framework (See Thomson 1994).

In a bargaining problem, efficiency of a utility vector is defined by Pareto optimality. We use weak Pareto optimality, in which an allocation is only Pareto improving if it increases the payoff of every agent.

Definition 2 For a bargaining problem $(S, d)$, an allocation $u \in S$ is Pareto optimal if and only if there is no $w \in S$ such that $w_{i}>u_{i}$ for all $i \in N$. The Pareto frontier, $P(S)$, is the set of Pareto optimal allocations:

$$
P(S) \equiv\left\{u \in S \mid \forall w \in S, \exists i \in N \text { s.t. } w_{i} \leq u_{i}\right\} .
$$

We use the KS solution concept as our bargaining solution in this model. This solution proceeds by identifying each agent's ideal (or maximal) payoff, $\bar{u}_{i}$. The solution is then the unique point where the ray between the threat point $d$ and ideal point $\bar{u}$ intersects the Pareto frontier. Note that the vector $\bar{u}$ often may not be feasible; if it is, the bargaining solution trivially selects it. Since we set $d=0$, this is formally defined as follows.

Definition 3 For a bargaining set $S \in \mathbb{R}_{+}^{n}$, let $\bar{u}_{i}=\max _{u \in S} u_{i}$ denote the ideal payoff of agent $i$. The Kalai-Smorodinsky solution is the unique point $u^{*} \in S$ such that $u^{*} \in P(S)$ and

$$
\frac{u_{i}}{\bar{u}_{i}}=\frac{u_{j}}{\bar{u}_{j}} \quad \text { for all } i, j \in N \text {. }
$$

Figure 1 also illustrates the KS solution for simple bargaining sets in both the two-person and three-person cases.

Our purpose in this paper is to evaluate how the KS solution is affected when individuals commit to unilateral monetary transfers prior to applying the bargaining solution. Indeed, we restrict those transfers to a particular form: agent $i$ commits to transfer fraction $\lambda_{i}$ of his utility to agent $i-1$.

Definition 4 A pre-donation is a vector $\lambda \in[0,1]^{n}$ where $\lambda_{1}=0$.

Definition 5 The distorted bargaining set for simple bargaining set $S_{\alpha}$ and pre-donation $\lambda$ is defined as:

$$
S_{\alpha}(\lambda) \equiv\left\{u \in \mathbb{R}_{+}^{n} \mid \exists \hat{u} \in S_{\alpha} \text { s.t. } \begin{array}{l}
u_{n}=\left(1-\lambda_{n}\right) \hat{u}_{n} \text { and } \\
u_{i}=\left(1-\lambda_{i}\right)\left(\hat{u}_{i}+\frac{\lambda_{i+1} u_{i+1}}{1-\lambda_{i+1}}\right), \forall i \leq n-1
\end{array}\right\} .
$$

One can interpret pre-donations as payments made via a numeriare good for which everyone has a marginal utility of 1 . Notice that the pre-donation is a commitment to transfer a percentage of future utility, rather than a specific quantity of utils. Moreover, 

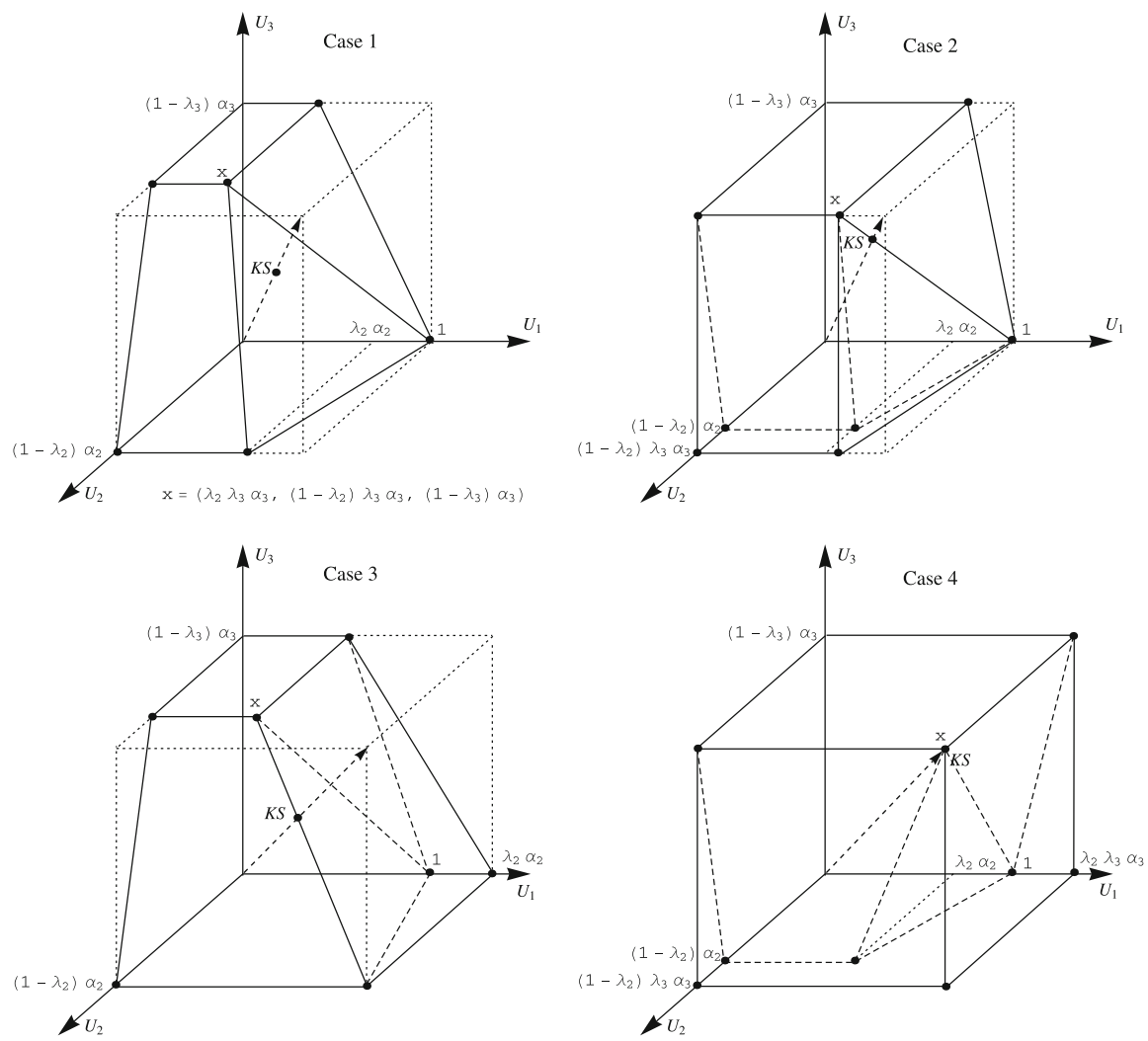

Fig. 2 The three-person bargaining set after pre-donations

percentage $\lambda_{i+1}$ applies to any pre-donation received by $i+1$, not just his utility from the allocation of the good. ${ }^{3}$

The pre-donation of agent $i$ has two important effects on the bargaining set. First, it reduces the maximal payoff of the donor, $\bar{u}_{i}$. Second, the pre-donations extend the Pareto frontier outward, since utility is transferred more efficiently between agents. ${ }^{4}$ These effects are illustrated in Fig. 2 for the three-agent game. Point $x$ in the graph lies strictly above the original set $S_{\alpha}$ because of the more efficient transfer of utility.

Our particular formulation restricts pre-donations in two ways. First, agents cannot pre-donate to those with a higher marginal utility, but it is never in agent $i$ 's interest to do so. An "upward" pre-donation is less efficient at transferring utility than simply

\footnotetext{
3 For this reason, there is no hat on $u_{i+1}$ in the definition. A transfer of $\lambda_{i+1} \hat{u}_{i+1}$ would only pass on a fraction of agent $i+1$ 's initial allocation of the good. If agent $i+1$ receives final utility $u_{i+1}$, he must have received $\frac{u_{i+1}}{1-\lambda_{i+1}} \geq \hat{u}_{i+1}$ before executing his pre-donation but after receiving any pre-donation from agent $i+2$. Agent $i$ is transferred fraction $\lambda_{i+1}$ of that utility.

${ }^{4}$ For example, to transfer one util from agent $i$ to agent $i-1$ by re-allocating the good, one would have to take $\frac{1}{\alpha_{i-1}}$ units of the good from $i$ and thus reduce his payoff by $\frac{\alpha_{i}}{\alpha_{i-1}}$ utils. To transfer one util by pre-donation would only reduce agent $i$ 's payoff by one util.
} 
allocating more of the good to the higher agent. Such a pre-donation would result in a utility vector that lies in the interior of the bargaining set. Moreover, it would reduce agent $i$ 's maximal payoff. Thus, he would always be awarded a smaller utility in the KS solution after such a pre-donation.

The more consequential restriction is that agents can only pre-donate to the agent whose marginal utility is immediately below his own (i.e. agent 3 cannot pre-donate directly to agent 1 , even if he desired to do so). However, he may indirectly donate to others, as agent 2 will pass along fraction $\lambda_{2}$ of whatever utility he receives, including agent 3's pre-donation. The trouble that arises with allowing a larger class of predonations is that donations to agent 1 become a public good. As agent 3 makes direct donations to 1 , agent 2 is able to profitably decrease his donations. We revisit this issue in the Conclusion.

When pre-donations are particularly large, then some of the boundaries of the set $S_{\alpha}(\lambda)$ will not even be weakly Pareto optimal. This occurs when the pre-donation is so large that an agent gets more utility when the object is fully allocated to his donor, rather than having any part of the object allocated directly to himself. This is illustrated for the three person case in Cases 2 through 4 of Fig. 2; the dashed lines represent the boundaries of the inefficient faces of $S_{\alpha}(\lambda)$. For this reason, we consider the comprehensive closure of the distorted bargaining set. This enlarges the bargaining set to include any point that offers less than or equal utility than some point in the distorted bargaining set.

Definition 6 The comprehensive closure of the distorted bargaining set is:

$$
\underset{\sim}{S_{\alpha}}(\lambda) \equiv\left\{u \in \mathbb{R}_{+}^{n} \mid \exists \hat{u} \in S_{\alpha}(\lambda) \text { s.t. } u_{i} \leq \hat{u}_{i} \forall i\right\}
$$

The solid lines in Fig. 2 denote the boundaries of $S_{\alpha}(\lambda)$. One can interpret the comprehensive closure as allowing the free disposal of utility. For instance, in Case 4, the point $\left(\lambda_{2} \lambda_{3} \alpha_{3},\left(1-\lambda_{2}\right) \lambda_{3} \alpha_{3}, 0\right)$ in the bottom-front-right corner is obtained by allocating the good fully to agent 3 , producing utility vector $\left(\lambda_{2} \lambda_{3} \alpha_{3},\left(1-\lambda_{2}\right) \lambda_{3} \alpha_{3}\right.$, $\left.\left(1-\lambda_{3}\right) \alpha_{3}\right)$, but then disposing of all agent 3's remaining utility. Note that the boundary of $\underset{\sim}{S_{\alpha}}(\lambda)$ is always weakly Pareto optimal. We will use the comprehensive closure in our analysis of the distorted game, but this is only a matter of analytical convenience; in equilibrium, the KS solution is the same for set $S_{\alpha}(\lambda)$ or set $\underset{\sim}{S_{\alpha}}(\lambda)$.

In our framework, agents simultaneously choose their pre-donations; then, without any further choices from agents, the KS solution is applied to the distorted bargaining set and actual transfers are realized according to the pre-donations. Consistent with a Nash equilibrium, we require that each agent's pre-donation $\lambda_{i}^{*}$ be a best response, taking all others' pre-donations $\lambda_{-i}^{*}$ as given.

Definition 7 A pre-donation equilibrium is a pre-donation $\lambda^{*}$ and allocation $u^{*}$ such that $u^{*}=K S\left(\underset{\sim}{S_{\alpha}}\left(\lambda^{*}\right)\right)$ and for all $i$ and all $\hat{\lambda}_{i} \in[0,1], u_{i}^{*} \geq K S_{i}\left({\underset{\sim}{\alpha}}_{\alpha}\left(\lambda_{-i}^{*}, \hat{\lambda}_{i}\right)\right)$. 


\section{A three-person bargaining problem}

We begin by examining this environment when there are exactly three agents. This offers a clear visualization of the pre-donations and the resulting KS solution, and makes clear the mechanics by which pre-donations achieve Pareto improvements. Also, we can fully characterize all the Nash equilibria in this environment; in the following section, we will restrict our attention to a few of these.

Consider a three-person simple bargaining problem where the bargaining set is:

$$
S_{\alpha}=\left\{u \in \mathbb{R}_{+}^{3} \mid u_{3} \leq \alpha_{3}, u_{2} \leq \alpha_{2}\left(1-\frac{u_{3}}{\alpha_{3}}\right), u_{1} \leq 1-\frac{u_{2}}{\alpha_{2}}-\frac{u_{3}}{\alpha_{3}}\right\},
$$

where $1 \leq \alpha_{2} \leq \alpha_{3}$. This set is a tetrahedron with the disagreement point fixed at the origin, as shown in Fig. 1. The Pareto frontier of $S_{\alpha}, P\left(S_{\alpha}\right)$, is described as the plane $u_{1}+\frac{u_{2}}{\alpha_{2}}+\frac{u_{3}}{\alpha_{3}}=1$. The undistorted KS solution selects the point on the frontier satisfying $u_{1}=\frac{u_{2}}{\alpha_{2}}=\frac{u_{3}}{\alpha_{3}}$, which is $K S\left(S_{\alpha}\right)=\left(\frac{1}{3}, \frac{\alpha_{2}}{3}, \frac{\alpha_{3}}{3}\right)$. In other words, the good is equally divided among the three (even though their final utilities differ).

While pre-donations always distort the bargaining set, the exact effect depends on the size of the pre-donation relative to the original maximal payoffs and pre-donations of others. In particular, we need to check whether a pre-donation to an agent is greater than that agent's utility from an outright allocation of the entire good; i.e. is $\lambda_{2} \alpha_{2}>1, \lambda_{3} \alpha_{3}>\alpha_{2}$, and/or $\lambda_{2} \lambda_{3} \alpha_{3}>1$.

This creates 8 possible configurations of these three inequalities. Two of these are self contradictory: if $\lambda_{2} \alpha_{2}>1$ and $\lambda_{3} \alpha_{3}>\alpha_{2}$ then we cannot have $\lambda_{2} \lambda_{3} \alpha_{3} \leq 1$, and similarly if all three inequalities are reversed. The remaining possibilities are examined below and illustrated in Fig. 2. Note that Cases 3 and 4 each include two of the eight possible configurations.

\subsection{Case 1: $\lambda_{2} \alpha_{2} \leq 1, \lambda_{3} \alpha_{3} \leq \alpha_{2}$, and $\lambda_{2} \lambda_{3} \alpha_{3} \leq 1$}

When $\lambda_{2} \alpha_{2} \leq 1$, and $\lambda_{3} \alpha_{3} \leq \alpha_{2}$ (as is the case in Fig. 2), the Pareto frontier is the convex hull of the points $(1,0,0),\left(\lambda_{2} \alpha_{2},\left(1-\lambda_{2}\right) \alpha_{2}, 0\right)$ and $\left(\lambda_{2} \lambda_{3} \alpha_{3},\left(1-\lambda_{2}\right) \lambda_{3} \alpha_{3}\right.$, $\left(1-\lambda_{3}\right) \alpha_{3}$ ), each of which results from allocating the good entirely to agent 1,2 , or 3 , respectively, and transferring utility as promised. Any convex combination of these points represents a particular division of the good among the agents. This convex hull is part of a plane, described by $u_{1}+\frac{1-\lambda_{2} \alpha_{2}}{\left(1-\lambda_{2}\right) \alpha_{2}} u_{2}+\frac{\alpha_{2}-\lambda_{3} \alpha_{3}}{\left(1-\lambda_{3}\right) \alpha_{2} \alpha_{3}} u_{3}=1$.

Notice that the maximal payoffs are now $1,\left(1-\lambda_{2}\right) \alpha_{2}$ and $\left(1-\lambda_{3}\right) \alpha_{3}$, respectively. Thus, the KS solution selects the point on the frontier such that $u_{1}=\frac{u_{2}}{\left(1-\lambda_{2}\right) \alpha_{2}}=$ $\frac{u_{3}}{\left(1-\lambda_{3}\right) \alpha_{3}}$. This creates a system of equations that has the solution:

$$
K S\left(S_{\alpha}, \lambda\right)=\left(\frac{\alpha_{2}}{3 \alpha_{2}-\lambda_{2} \alpha_{2}^{2}-\lambda_{3} \alpha_{3}}, \frac{\left(1-\lambda_{2}\right) \alpha_{2}^{2}}{3 \alpha_{2}-\lambda_{2} \alpha_{2}^{2}-\lambda_{3} \alpha_{3}}, \frac{\left(1-\lambda_{3}\right) \alpha_{2} \alpha_{3}}{3 \alpha_{2}-\lambda_{2} \alpha_{2}^{2}-\lambda_{3} \alpha_{3}}\right) .
$$


Note that $K S_{2}$ is increasing $\lambda_{2}$ iff $\alpha_{2}\left(\alpha_{2}-3\right)+\lambda_{3} \alpha_{3}>0$, and $K S_{3}$ is increasing $\lambda_{3}$ iff $\alpha_{2}\left(\lambda_{2} \alpha_{2}-3\right)+\alpha_{3}>0$.

One can readily show that when $\lambda_{2} \leq \frac{1}{\alpha_{2}}$ and $\lambda_{3} \leq \frac{\alpha_{2}}{\alpha_{3}}$, this solution lies in the convex hull previously described, which is to say that the ray from the origin always intersects the triangular face shown in Case 1 of Fig. 2, rather than one of the other faces.

\subsection{Case 2: $\lambda_{2} \alpha_{2} \leq 1, \lambda_{3} \alpha_{3}>\alpha_{2}$, and $\lambda_{2} \lambda_{3} \alpha_{3} \leq 1$}

When $\lambda_{3} \alpha_{3}>\alpha_{2}$, agent 2 gains more when the good is allocated to agent 3 than when it is allocated to himself. Thus, any point in $S_{\alpha}(\lambda)$ which partially allocates the good to agent 2 (on the front-facing boundaries) is inefficient, and the comprehensive closure will expand the bargaining set to $S_{\alpha}(\lambda)$ as shown in Fig. 2.

In particular, the triangular face described in Case 1 is now replaced by the convex hull of $(1,0,0),\left(\lambda_{2} \lambda_{3} \alpha_{3},\left(1-\lambda_{2}\right) \lambda_{3} \alpha_{3}, 0\right)$ and $\left(\lambda_{2} \lambda_{3} \alpha_{3},\left(1-\lambda_{2}\right) \lambda_{3} \alpha_{3},\left(1-\lambda_{3}\right) \alpha_{3}\right)$, which is depicted by the plane $u_{1}+\frac{1-\lambda_{2} \lambda_{3} \alpha_{3}}{\left(1-\lambda_{2}\right) \lambda_{3} \alpha_{3}} u_{2}=1$.

The maximal payoff of 2 is now $\left(1-\lambda_{2}\right) \lambda_{3} \alpha_{3}$, so the KS solution selects the point on the frontier where $u_{1}=\frac{u_{2}}{\left(1-\lambda_{2}\right) \lambda_{3} \alpha_{3}}=\frac{u_{3}}{\left(1-\lambda_{3}\right) \alpha_{3}}$. This system of equations has the solution:

$$
K S\left(S_{\alpha}, \lambda\right)=\left(\frac{1}{2-\lambda_{2} \lambda_{3} \alpha_{3}}, \frac{\left(1-\lambda_{2}\right) \lambda_{3} \alpha_{3}}{2-\lambda_{2} \lambda_{3} \alpha_{3}}, \frac{\left(1-\lambda_{3}\right) \alpha_{3}}{2-\lambda_{2} \lambda_{3} \alpha_{3}}\right)
$$

It is noteworthy that this solution always lies on the line segment between $(1,0,0)$ and $\left(\lambda_{2} \lambda_{3} \alpha_{3},\left(1-\lambda_{2}\right) \lambda_{3} \alpha_{3},\left(1-\lambda_{3}\right) \alpha_{3}\right)$, which is feasible in both $S_{\alpha}(\lambda)$ and $S_{\alpha}(\lambda)$; indeed, the KS solution would be the same under either.

Note that $K S_{2}$ is increasing $\lambda_{2}$ iff $\alpha_{3} \lambda_{3}>2$, and $K S_{3}$ is increasing $\lambda_{3}$ iff $\alpha_{3} \lambda_{2}>2$.

\subsection{Case 3: $\lambda_{2} \alpha_{2}>1, \lambda_{3} \alpha_{3} \leq \alpha_{2}$, and $\lambda_{2} \lambda_{3} \alpha_{3} \lessgtr 1$}

When $\lambda_{2} \alpha_{2}>1$, agent 1 benefits more when agent 2 is allocated the entire good than when agent 1 is. However, since $\lambda_{3} \alpha_{3} \leq \alpha_{2}$, agent 1 gets more in pre-donations when agent 2 is allocated the good than when agent 3 is. Thus, any point in $S_{\alpha}(\lambda)$ which partially allocates the good to agent 1 (on the right-facing boundaries) is inefficient, and the comprehensive closure will expand the bargaining set to $S_{\alpha}(\lambda)$ as shown in Fig. 2. The relevant face of $\underset{\sim}{S_{\alpha}}(\lambda)$ is described by $u_{1}+\frac{\lambda_{2}\left(\alpha_{2}-\lambda_{3} \alpha_{3}\right)}{\left(1-\lambda_{3}\right) \alpha_{3}} u_{3}=\lambda_{2} \alpha_{2}$.

After adjusting maximal payoffs, the KS solution selects the point on the frontier where $\frac{u_{1}}{\lambda_{2} \alpha_{2}}=\frac{u_{2}}{\left(1-\lambda_{2}\right) \alpha_{2}}=\frac{u_{3}}{\left(1-\lambda_{3}\right) \alpha_{3}}$. This system of equations has the solution:

$$
K S\left(S_{\alpha}, \lambda\right)=\left(\frac{\lambda_{2} \alpha_{2}}{1+\lambda_{2}\left(\alpha_{2}-\lambda_{3} \alpha_{3}\right)}, \frac{\left(1-\lambda_{2}\right) \alpha_{2}}{1+\lambda_{2}\left(\alpha_{2}-\lambda_{3} \alpha_{3}\right)}, \frac{\left(1-\lambda_{3}\right) \lambda_{2} \alpha_{2} \alpha_{3}}{1+\lambda_{2}\left(\alpha_{2}-\lambda_{3} \alpha_{3}\right)}\right) .
$$


Table 1 Equilibrium pre-donations $\left(\lambda_{2}^{*}, \lambda_{3}^{*}\right)$ in the three-person bargaining game

\begin{tabular}{llll}
\hline & $\alpha_{3} \leq 2 \alpha_{2}$ & $2 \alpha_{2} \leq \alpha_{3} \leq 3 \alpha_{2}$ & $\alpha_{3} \geq 3 \alpha_{2}$ \\
\hline$\alpha_{2} \leq 2$ & $(0,0)$ & $(0,0)$ & $\left(0, \frac{\alpha_{2}}{\alpha_{3}}\right)$ \\
$2 \leq \alpha_{2} \leq 3$ & $(0,0)$ & $(0,0)\left(\frac{3 \alpha_{2}-\alpha_{3}}{\alpha_{2}^{2}}, \frac{\left(3-\alpha_{2}\right) \alpha_{2}}{\alpha_{3}}\right)\left(\frac{1}{\alpha_{2}}, \frac{\alpha_{2}}{\alpha_{3}}\right)$ & $\left(\frac{1}{\alpha_{2}}, \frac{\alpha_{2}}{\alpha_{3}}\right)$ \\
$\alpha_{2} \geq 3$ & $\left(\frac{1}{\alpha_{2}}, 0\right)$ & $\left(\frac{1}{\alpha_{2}}, \frac{\alpha_{2}}{\alpha_{3}}\right)$ & $\left(\frac{1}{\alpha_{2}}, \frac{\alpha_{2}}{\alpha_{3}}\right)$ \\
$\alpha_{2} \leq 2$ and $\alpha_{3} \geq 4$ & & $\lambda_{2}^{*} \in\left[\frac{2}{\alpha_{3}}, \frac{1}{2}\right]$ and $\lambda_{3}^{*}=\frac{2}{\alpha_{3}}$ & \\
\hline
\end{tabular}

Table 2 Equilibrium payoffs in the three-person bargaining game

\begin{tabular}{llll}
\hline$\left(\lambda_{2}^{*}, \lambda_{3}^{*}\right)$ & $u_{1}^{*}$ & $u_{2}^{*}$ & $u_{3}^{*}$ \\
\hline$(0,0)$ & $\frac{1}{3}$ & $\frac{\alpha_{2}}{3}$ & $\frac{\alpha_{3}}{3}$ \\
$\left(\frac{1}{\alpha_{2}}, 0\right)$ & $\frac{1}{2}$ & $\frac{\alpha_{2}-1}{2}$ & $\frac{\alpha_{3}}{2}$ \\
$\left(0, \frac{\alpha_{2}}{\alpha_{3}}\right)$ & $\frac{1}{2}$ & $\frac{\alpha_{2}}{2}$ & $\frac{\alpha_{3}-\alpha_{2}}{2}$ \\
$\left(\frac{1}{\alpha_{2}}, \frac{\alpha_{2}}{\alpha_{3}}\right)$ & 1 & $\alpha_{2}-1$ & $\alpha_{3}-\alpha_{2}$ \\
$\left(\frac{3 \alpha_{2}-\alpha_{3}}{\alpha_{2}^{2}}, \frac{\left(3-\alpha_{2}\right) \alpha_{2}}{\alpha_{3}}\right)$ & $\frac{\alpha_{2}}{\alpha_{3}-\alpha_{2}\left(3-\alpha_{2}\right)}$ & 1 & $\alpha_{2}$ \\
$\left(\lambda_{2}^{*}, \frac{2}{\alpha_{3}}\right)$ & $\frac{1}{2\left(1-\lambda_{2}^{*}\right)}$ & 1 & $\frac{\alpha_{3}-2}{2\left(1-\lambda_{2}^{*}\right)}$ \\
\hline
\end{tabular}

This solution always lies on the line segment between $\left(\lambda_{2} \lambda_{3} \alpha_{3},\left(1-\lambda_{2}\right) \lambda_{3} \alpha_{3}\right.$, $\left.\left(1-\lambda_{3}\right) \alpha_{3}\right)$ and $\left(\lambda_{2} \alpha_{2},\left(1-\lambda_{2}\right) \alpha_{2}, 0\right)$, and is thus the KS solution under both $S_{\alpha}(\lambda)$ and $S_{\alpha}(\lambda)$. Also, note that $K S_{2}$ is increasing $\lambda_{2}$ iff $\alpha_{3} \lambda_{3}>1+\alpha_{2}$ (which contradicts $\lambda_{3} \alpha_{3} \leq \alpha_{2}$ ), and $K S_{3}$ is increasing $\lambda_{3}$ iff $\alpha_{3} \lambda_{2}>1+\lambda_{2} \alpha_{2}$.

\subsection{Case 4: $\lambda_{2} \alpha_{2} \lessgtr 1, \lambda_{3} \alpha_{3}>\alpha_{2}$ and $\lambda_{2} \lambda_{3} \alpha_{3}>1$}

If $\lambda_{3} \alpha_{3}>\alpha_{2}$ and $\lambda_{2} \lambda_{3} \alpha_{3}>1$, then donations from agent 3 are strictly better for agent 2 and agent 1 than for either of them to be directly allocated a portion of the good. Thus, $S_{\alpha}(\lambda)$ becomes a rectangle, and the KS solution will be its upper corner, $x=\left(\lambda_{2} \lambda_{3} \alpha_{3},\left(1-\lambda_{2}\right) \lambda_{3} \alpha_{3},\left(1-\lambda_{3}\right) \alpha_{3}\right)$. Indeed, we would obtain the same result applying the KS solution to $S_{\alpha}(\lambda)$. Note that $K S_{2}$ and $K S_{3}$ are strictly declining in $\lambda_{2}$ and $\lambda_{3}$, respectively.

\subsection{Pre-donation equilibria}

The equilibria of the pre-donation game depend on the original maximal payoffs, $\alpha_{i}$. All of the Nash equilibria of the pre-donation game are reported in Table 1, and the associated KS payoff is given in Table 2. These are found by checking the first order conditions of the various cases; we now illustrate this for two of the most important cases. 
First, suppose that $\alpha_{2} \leq 3$ and $\alpha_{3} \leq 3 \alpha_{2}$. We can verify that $\lambda_{2}^{*}=\lambda_{3}^{*}=0$ constitute a pre-donation equilibrium. Note that Case 1 applies, and thus both agent 2 and agent 3 are harmed by any increase in pre-donations. This outcome is, of course, identical to the non-distorted KS solution.

Next, suppose that $\alpha_{2} \geq 2$ and $\alpha_{3} \geq 2 \alpha_{2}$, and consider $\lambda_{2}^{*}=\frac{1}{\alpha_{2}}$ and $\lambda_{3}^{*}=\frac{\alpha_{2}}{\alpha_{3}}$. Again, Case 1 applies, and if $\alpha_{2} \geq 2$ and $\alpha_{3} \geq 2 \alpha_{2}$, then both agent 2 and agent 3 would be harmed by any decrease in pre-donations. If Agent 2 increases his pre-donation further, Case 3 applies and his payoff strictly decreases. If Agent 3 increases $\lambda_{3}$, Case 4 applies and his payoff strictly decreases. This equilibrium results in payoffs $\left(1, \alpha_{2}-1, \alpha_{3}-\alpha_{2}\right)$.

Note that whenever a pre-donation occurs in any equilibrium, it is Pareto-improving over the KS solution without pre-donations. Also, notice that that for certain parameter values (when $2 \leq \alpha_{2} \leq 3$ and $2 \alpha_{2} \leq \alpha_{3} \leq 3 \alpha_{2}$, or when $\alpha_{2} \leq 2$ and $\alpha_{3} \geq 4$ ), multiple equilibria exist. This should not be particularly surprising, since agent 2's donations are made more beneficial by agent 3's donations, and vice versa. Thus, each could abstain because the other is abstaining. The multiple equilibria indicate a potential coordination problem among the donors.

\section{The $n$-person bargaining problem}

In this section we characterize two of the equilibria of the $n$-person simple bargaining problem: one in which everyone makes pre-donations, and one in which no one does. Of course, other equilibria can arise, as exemplified in the three-person game from the preceding section; indeed, for some parameter values, neither of the two equilibria described here exist. However, the process for finding other equilibria is well-exemplified by the three-person case; and deriving this in full generality would require an analysis of $2^{n}$ cases.

We first turn to the equilibrium in which all agents are willing to make pre-donations. Theorem 1 establishes that each bargainer has incentive to distort the bargaining set when his marginal utility is sufficiently large, relative to the agent immediately below him.

Theorem 1 Whenever $\alpha_{i} \geq 2 \alpha_{i-1}$ for all $i \in N \backslash\{1\}$, then $\lambda_{i}^{*}=\frac{\alpha_{i-1}}{\alpha_{i}}$ and $u_{i}^{*}=$ $\alpha_{i}-\alpha_{i-1}$ for all $i \in N \backslash\{1\}$ and $u_{1}^{*}=1$ constitutes a pre-donation equilibrium. Furthermore, if the inequality is strict for all $i$, this outcome is strongly Pareto improving over the undistorted KS solution.

Proof We begin by examining the analog to Case 1 in the previous section, where $\lambda_{i} \leq \frac{\alpha_{i-1}}{\alpha_{i}}$ for all $i$. To determine the relevant hyperplane, consider the outcome of various feasible allocations. If the good were fully allocated to agent $i$, after pre-donations were executed agent $i$ would receive $u_{i}=\left(1-\lambda_{i}\right) \alpha_{i}$, while agents $j>i$ receive $u_{j}=0$ and agents $j<i$ receive $u_{j}=\left(1-\lambda_{j}\right) \alpha_{i} \prod_{k=j+1}^{i} \lambda_{k}$.

If this calculation is repeated, once for each agent fully receiving the good, it produces $n$ utility vectors; all other efficient allocations are merely a convex combination 
of these. This efficient frontier can be described by the hyperplane:

$$
u_{1}+\sum_{j=2}^{n} \frac{\alpha_{j-1}-\lambda_{j} \alpha_{j}}{\left(1-\lambda_{j}\right) \alpha_{j-1} \alpha_{j}} u_{j}=1
$$

The assumption that $\lambda_{i} \leq \frac{\alpha_{i-1}}{\alpha_{i}}$ ensures that the coefficient of $u_{i}$ is positive for all $i$.

The KS Solution also requires that $\frac{u_{i}}{\left(1-\lambda_{i}\right) \alpha_{i}}=\frac{u_{j}}{\left(1-\lambda_{j}\right) \alpha_{j}}$. Thus, we can substitute for $u_{j}$ with $\frac{\left(1-\lambda_{j}\right) \alpha_{j}}{\left(1-\lambda_{i}\right) \alpha_{i}} u_{i}$ for all $j \neq i$ and find the final utility awarded to $i$ (after pre-donations are executed) in the KS solution :

$$
K S_{i}\left(\lambda_{-i}, \lambda_{i}\right)=\frac{\left(1-\lambda_{i}\right) \alpha_{i}}{1+\sum_{j=2}^{n} \frac{\alpha_{j-1}-\lambda_{j} \alpha_{j}}{\alpha_{j-1}}}
$$

It is a small algebraic exercise to show that this solution is in fact a convex combination of the $n$ vectors (rather than lying on the hyperplane but outside the convex hull).

Now consider possible deviations of agent $i$, taking as given $\lambda_{-i}^{*}$. If $i$ plays $\lambda_{i}<$ $\frac{\alpha_{i-1}}{\alpha_{i}}$, his final payoff is: $K S_{i}\left(\lambda_{-i}^{*}, \lambda_{i}\right)=\frac{\left(1-\lambda_{i}\right) \alpha_{i-1} \alpha_{i}}{2 \alpha_{i-1}-\lambda_{i} \alpha_{i}}$. The derivative $\frac{\partial K S_{i}}{\partial \lambda_{i}}$ is positive if and only if $\alpha_{i} \geq 2 \alpha_{i-1}$. Thus, agent $i$ never has an incentive to reduce his pre-donation.

If $i$ plays $\lambda_{i}>\frac{\alpha_{i-1}}{\alpha_{i}}$, we no longer are in the equivalent of Case 1. Instead, given $\lambda_{-i}^{*}$, it resembles Case 4. Specifically, agents 1 through $i-1$ receive higher utility if the good is allocated to any of agents $i$ through $n$ rather than to any combination of agents 1 through $i-1$, and thus such allocations are inefficient. Formally, $\left(1-\lambda_{j}\right) \alpha_{j}<\left(1-\lambda_{j}\right) \alpha_{i} \prod_{k=j+1}^{i} \lambda_{k}$ for all $j<i$. Moreover, fully allocating the good to agent $j \in\{i, \ldots n-1\}$ is weakly inefficient; if it were awarded instead to agent $n$, any agents at or below $j$ would receive the same utility, yet agents $m>j$ would jump from 0 to $u_{m}=\left(1-\lambda_{m}\right) \alpha_{n} \prod_{k=m+1}^{i} \lambda_{k}$.

Thus, when $\lambda_{i}>\frac{\alpha_{i-1}}{\alpha_{i}}$, the bargaining set is an $n$-dimensional box, and the KS solution will select its corner, where $u_{m}=\alpha_{m}-\alpha_{m-1}$ for all $m \in\{i+1, \ldots, n\}, u_{i}=$ $\left(1-\lambda_{i}\right) \alpha_{i}, u_{j}=\left(\alpha_{j}-\alpha_{j-1}\right) \frac{\alpha_{i}}{\alpha_{i-1}} \lambda_{i}$ for all $j \in\{2, \ldots, i-1\}$, and $u_{1}=\frac{\alpha_{i}}{\alpha_{i-1}} \lambda_{i}$. Hence increasing $\lambda_{i}$ strictly reduces the final utility awarded to agent $i$ after the execution of the KS solution and pre-donations.

Thus, $\lambda_{i}^{*}=\frac{\alpha_{i-1}}{\alpha_{i}}$ is a best response to $\lambda_{-i}^{*}$. This is a Pareto improvement over the undistorted KS solution, where each agent $i$ would have been awarded $u_{i}=\frac{\alpha_{i}}{n}$. If $\alpha_{i}>2 \alpha_{i-1}$, then $\alpha_{i}+(n-2) \alpha_{i-1}>n \alpha_{i-1}$, and $(n-1) \alpha_{i}>n \alpha_{i-1}$. Thus $\alpha_{i}-\alpha_{i-1}>\frac{\alpha_{i}}{n}$.

Of course, to reach this outcome, agents' marginal utilities must vary significantly, each being at least twice as great as the preceding one. While this is a strong condition, it could nonetheless be plausible. For instance, a homogenous good is often sold for a wide range of prices across various retailers. This price dispersion can be supported in equilibrium due to underlying differences in consumers' locations, preferences, or search costs. Akin and Platt (2008) document remarkable price dispersion in commonly-used prescription drugs, using US micro-data. For example, a 30-day supply of $10 \mathrm{mg}$ Lipitor pills (the most commonly prescribed medicine in the US) was 
purchased at prices ranging from $\$ 52.6$ to $\$ 236.6$. They explain this price dispersion in a search model with insurance.

If $\alpha_{i} \geq 2 \alpha_{i-1}$ only holds for some of the agents, they may still engage in predonations, despite the non participation of others. This would give rise to equilibria similar to those in the previous section, where only agent 2 or only agent 3 made pre-donations.

There are also equilibria in which no pre-donations occur. This equilibrium exists when each agent has a marginal utility that is sufficiently small, relative to the agent immediately below him. On the other hand, if marginal utilities are sufficiently distinct for at least one pair of agents $\left(\alpha_{i}>n \alpha_{i-1}\right.$ for some $\left.i\right)$, some pre-donations must occur in equilibrium.

Theorem $2 \lambda_{i}^{*}=0$ and $u_{i}^{*}=\frac{\alpha_{i}}{n}$ for all $i \in N$ constitutes a pre-donation equilibrium if and only if $\alpha_{i} \leq n \alpha_{i-1}$ for all $i \in N \backslash\{1\}$.

Proof Suppose all agents except $i$ set $\lambda_{-i}^{*}=0$. This places us within the analog to Case 1, and the KS solution in Eq. 4 applies. Thus, $K S_{i}\left(\lambda_{-i}^{*}, \lambda_{i}\right)=\frac{\left(1-\lambda_{i}\right) \alpha_{i-1} \alpha_{i}}{n \alpha_{i-1}-\lambda_{i} \alpha_{i}}$. The derivative $\frac{\partial K S_{i}}{\partial \lambda_{i}}$ is negative if and only if $\alpha_{i} \leq n \alpha_{i-1}$. Thus $\lambda_{i}=0$ is a best response if this holds; but if $\alpha_{i}>n \alpha_{i-1}, i$ will make a pre-donation even if all others abstain.

Note the overlap between the conditions of Theorems 1 and 2, indicating that in intermediate ranges $\left(2 \alpha_{i-1} \leq \alpha_{i} \leq n \alpha_{i-1}\right.$ for all $i$ ), both equilibria exist. In such circumstances, coordination would be necessary to reach the Pareto-superior equilibrium that has positive pre-donations.

\section{Relation to division problems}

In our original simple bargaining problem, the question is how to allocate a divisible good among bargainers who have distinct but constant marginal utilities from the good. By introducing pre-donations, we allow agents to directly transfer utility via some numeraire good, although such transfers are voluntary and thus only occur if they are individually rational.

An alternative but related approach is to frame this as a division problem, as was done for the two-person case in Sertel $(1991,1992)$. Sometimes called a bankruptcy problem, the division problem is a question of how to split $E$ dollars among $n$ agents when the sum of their individual claims, $\sum_{i} \alpha_{i}$, is greater than $E$. Any prescribed resolution to the problem is called a division rule, which Thomson (2003) defines ${ }^{5}$ as a function $D: \mathbb{R}_{+}^{n+1} \rightarrow \mathbb{R}_{+}^{n}$ that splits the available amount such that no agent can receive more than his claim, $D_{i}(E, \alpha) \in\left[0, \alpha_{i}\right]$, and the sum of the payoffs should be equal to the available amount, $\sum_{i=1}^{n} D_{i}(E, \alpha)=E$.

In translating our environment to a division problem, we set $E=\alpha_{n}$ and refer to marginal utilities $\alpha_{i}$ as claims. Thus, it is as if the object is allocated entirely to agent $n$ (who values it the most), after which the division rule dictates how large of transfers

\footnotetext{
5 See also Moulin (2002).
} 
Table 3 Applications of the concessionary division rule in the three-person case

\begin{tabular}{llllll}
\hline$\alpha$ & $(1,3,6)$ & $(1,3,4)$ & $(1,2,4)$ & $\left(1, \frac{3}{2}, 4\right)$ & $\left(1, \frac{3}{2}, 2\right)$ \\
$C D$ & $(1,2,3)$ & $\left(1, \frac{3}{2}, \frac{3}{2}\right)$ & $(1,1,2)$ & $\left(\frac{3}{4}, \frac{3}{4}, \frac{5}{2}\right)$ & $\left(\frac{2}{3}, \frac{2}{3}, \frac{2}{3}\right)$ \\
\hline
\end{tabular}

$n$ must give to each of the other agents. Unlike a pre-donation, these transfers are imposed, rather than chosen by the individual agent.

We now consider the relationship between our pre-donation equilibrium and division rules. To some extent, these can never perfectly align. In an equilibrium where some agent abstains from pre-donating, the resulting payoffs will sum to something strictly less than $\alpha_{n}$-precisely because the KS solution still allocates a portion of the good to someone who values it less than $\alpha_{n}$. This outcome, by definition, is never contemplated in a division rule.

However, the equilibrium where $\lambda_{i}^{*}=\frac{\alpha_{i-1}}{\alpha_{i}}$ for all $i$ perfectly aligns with the concessionary division rule. For the two-person division problem, this was coined by Sertel (1991) as follows: Agent 2 concedes to agent 1 the highest payoff possible (up to agent 1's claim), subject to the condition that agent 2 gets no less than agent 1.

In practice, this rule would assign agent 1 a payoff of $\frac{\alpha_{2}}{2}$, unless this exceeded his claim, $\alpha_{1}=1$. Thus, the payoff to agent 1 is $C D_{1}=\min \left\{1, \frac{\alpha_{2}}{2}\right\}$, while agent 2 receives $C D_{2}=\alpha_{2}-C D_{1}$. For instance, claims of $(1,1.5)$ result in payoffs $(0.75,0.75)$, while claims of $(1,3)$ generate payoffs $(1,2)$.

We generalize the concessionary division rule to the $n$-person case as follows:

Definition 8 For any vector of claims $\alpha \equiv\left(\alpha_{i}\right)_{i \in N}$, with value to be divided $E=\alpha_{n}$, let the concession of each agent $i \in N$ be defined as:

$$
C_{i} \equiv \min _{j \in\{1, \ldots, i\}} \frac{(j-1) C_{i+1}+\alpha_{i-j}}{j},
$$

with $C_{n+1} \equiv \alpha_{n}$ and $\alpha_{0} \equiv 0$. The concessionary division rule assigns agent $i$ a payoff of $C D_{i}\left(\alpha_{n}, \alpha\right) \equiv C_{i+1}-C_{i}$.

Under this rule, it is as if each agent $i$ receives his full claim $\alpha_{i}$, but then concedes the full claim $\alpha_{i-1}$ to the agent immediately below him, giving him a net payoff of $\alpha_{i}-\alpha_{i-1}$. In the computation of concessions $C_{i}$, this occurs when $j=1$ is the minimizer for all $i$. This is subject to a caveat, however, that an agent with a higher claim should never receive a smaller payoff than agents with lower claims. When $j>1$ is the minimizer for some agent $i$, conceding the full amount $\alpha_{i-1}$ would leave $i$ 's payoff less than agent $i-j-1$ 's. Thus, his concession is reduced, resulting in an equal payoff for him and the $j-1$ agents below him. Table 3 provides a few examples in the three-person case.

Notice that if $\alpha_{i} \geq 2 \alpha_{i-1}$ for all $i$, then for any $j \in\{2, \ldots, i\},(j-1) \alpha_{i} \geq j \alpha_{i-1}>$ $j \alpha_{i-1}-\alpha_{i-j}$. Thus $\alpha_{i-1}<\frac{(j-1) \alpha_{i}+\alpha_{i-j}}{j}$, and the concessionary division rule will assign $C_{i}=\alpha_{i-1}$ and $C D_{i}=\alpha_{i}-\alpha_{i-1}$. This leads immediately to the following corollary. 
Corollary (to Theorem 1) When $\alpha_{i} \geq 2 \alpha_{i-1}$ for all $i$, the outcome of equilibrium pre-donations $\lambda_{i}^{*}=\frac{\alpha_{i-1}}{\alpha_{i}}$ coincides with the concessionary division rule.

\section{Conclusion}

In this paper we analyzed the distortion of the $n$-person KS bargaining solution via pre-donations. When agents are allowed to simultaneously make pre-donations to the agent whose marginal utility is immediately below their own, the equilibrium outcome will often Pareto dominate the undistorted KS solution. In particular, if marginal utilities are sufficiently distinct, the agent with the highest marginal utility is allocated the entire good but provides compensation to the others via pre-donations. In this case, the resulting payoffs coincide with the concessionary division rule.

Admittedly, our framework imposes an important restriction on pre-donations, in that agent $i$ can only pre-donate to agent $i-1$. We could instead consider a game where agents can directly pre-donate to anyone with a lower marginal utility; however, this model will not typically have an equilibrium in which all agents participate in pre-donation. For instance, suppose agent $n$ pre-donates to agent 1 . This distortion to the bargaining set also benefits agents 2 through $n-1$, regardless of whether they likewise pre-donate to agent 1 . As a consequence, pre-donations to agent 1 are like a public good, and these lower agents can free-ride on pre-donations from $n$.

Indeed, the best response of the lower agents will typically be to set their predonation to 0 when agent $n$ 's is positive. Moreover, this may not stop agent $n$ from pre-donating. A voluntary contribution model of public goods often results in a corner solution in which many agents contribute nothing, while the agent with the highest marginal value from the public good becomes its sole provider.

Another assumption we have maintained throughout is that marginal utilities strictly differ. This makes it unambiguous as to where pre-donations are directed. One could accommodate $\alpha_{i}=\alpha_{j}$ where $i>j$ for some agents by requiring that a pre-donation from agent $i+1$ be split equally among all such agents, and that pre-donations to agent $j-1$ come equally from all such agents. If the latter is not imposed, the preceding free rider problems would certainly arise. At the same time, one should note that even when $\alpha_{i}$ is close to $\alpha_{i-1}$, the equilibrium in Theorem 1 does not exist and agent $i$ will abstain from pre-donating.

Transfering of a share of future payoffs is a natural means by which bargainers can alter the allocation of a good when the solution concept and the bargaining set are public knowledge. As noted by Sertel (1992), no legal obstacle under commercial law can stop agents from signing contracts under which everybody will be better-off. Thus, individuals might generally manipulate many of the well-known axiomatic bargaining solutions, such as the Nash, Maschler-Perles, Egalitarian, and Utilitarian solutions.

\section{References}

Akin SN (2001) The n-person kalai-smorodinsky bargaining solution manipulated by pre-donations is concessionary. M.A. Thesis, Bogazici University, Istanbul, Turkey

Akin SN, Platt BC (2008) Search, moral hazard, and equilibrium price dispersion. University of Miami Working Paper 0718, FL, USA 
Atlamaz M, Klaus B (2007) Manipulation via endowments in exchange markets with indivisible goods. Soc Choice Welfare 28:1-18

Hurwicz L (1972) On informationally decentralized systems. In: McGuire CB, Radner R (eds) Decision and organization: a volume in honor of Jacob Marschak. North-Holland Publishers, Amsterdam

Kalai E, Smorodinsky M (1975) Other solutions to nash's bargaining problem. Econometrica 43:513-518

Kalai E (1977) Proportional solutions to bargaining situations: interpersonal utility comparisons. Econometrica 45:1623-1630

Kalai E (1985) Solutions to the bargaining problem. In: Hurwicz L, Schmeidler D, Sonnenschein H (eds) Social goals and organization. Cambridge University Press, Cambridge

Maschler M, Perles MA (1981) A super-additive solution for the nash bargaining game. Int J Game Theor 10:163-193

Moulin H (2002) Axiomatic cost and surplus sharing, Chapter 6, 289-357. In: Arrow KJ, Sen AK, Suzumura KHandbook of social choice and welfare, vol 1. Elsevier, Amsterdam

Nash JF (1950) The bargaining problem. Econometrica 28:155-162

Orbay BZ (2003) Kalai-Smorodinsky and Maschler-Perles solutions under pre-donation. In: Sertel M, Koray S Advances in economic design. Springer, Berlin

Postlewaite A (1974) Manipulation via endowments. Rev Econ Stud 46:255-263

Sertel MR (1991) Unpublished lecture notes on manipulation of the nash, Kalai-Smorodinsky, MaschlerPerles, egalitarian and utilitarian bargaining solutions, Bogazici University, Istanbul

Sertel MR (1992) The nash bargaining solution manipulated by pre-donations is talmudic. Econ Lett 40: 45-55

Sertel MR (1994) Manipulating lindahl equilibria via endowments. Econ Lett 46:167-171

Sertel MR, Orbay BZ (1998) Nash bargaining solution under pre-donations and collusion in a duopoly. METU Stud Dev 25:585-599

Sertel MR, Sanver I (2002) Manipulability of the men-(women)-optimal matching rule via endowments. Math Soc Sci 44:65-83

Thomson W (1994) Cooperative models of bargaining, Chapter 35, 1237-1284. In: Aumann R, Hart S (eds) Handbook of game theory, vol 2. Elsevier, Amsterdam

Thomson W (2003) Axiomatic and game-theoretic analysis of bankruptcy and taxation problems: a survey. Math Soc Sci 45:249-297 